\title{
Research Article \\ Some Opial Dynamic Inequalities Involving Higher Order Derivatives on Time Scales
}

\author{
Samir H. Saker \\ Department of Mathematics, Faculty of Science, Mansoura University, Mansoura 35516, Egypt \\ Correspondence should be addressed to Samir H. Saker, shsaker@mans.edu.eg
}

Received 25 April 2012; Accepted 16 August 2012

Academic Editor: Eric R. Kaufmann

Copyright (C 2012 Samir H. Saker. This is an open access article distributed under the Creative Commons Attribution License, which permits unrestricted use, distribution, and reproduction in any medium, provided the original work is properly cited.

We will prove some new Opial dynamic inequalities involving higher order derivatives on time scales. The results will be proved by making use of Hölder's inequality, a simple consequence of Keller's chain rule and Taylor monomials on time scales. Some continuous and discrete inequalities will be derived from our results as special cases.

\section{Introduction}

In the past decade a number of Opial dynamic inequalities have been established by some authors which are motivated by some applications; we refer to the papers [1-3]. The general idea is to prove a result for a dynamic inequality where the domain of the unknown function is a so-called time scale $\mathbb{T}$, which may be an arbitrary closed subset of the real numbers $\mathbb{R}$, to avoid proving results twice, once on a continuous time scale which leads to a differential inequality and once again on a discrete time scale which leads to a difference inequality. The three most popular examples of calculus on time scales are differential calculus, difference calculus, and quantum calculus (see [4]), that is, when $\mathbb{T}=\mathbb{R}, \mathbb{T}=\mathbb{N}$ and $\mathbb{T}=q^{\mathbb{N}_{0}}=\left\{q^{t}\right.$ : $\left.t \in \mathbb{N}_{0}\right\}$ where $q>1$. A cover story article in New Scientist [5] discusses several possible applications of time scales. In this paper, we will assume that sup $\mathbb{T}=\infty$ and define the time scale interval $[a, b]_{\mathbb{T}}$ by $[a, b]_{\mathbb{T}}:=[a, b] \cap \mathbb{T}$. Since the continuous and discrete inequalities involving higher order derivatives are important in the analysis of qualitative properties of solutions of differential and difference equations [6-8], we also believe that the dynamic inequalities involving higher order derivatives on time scales will play the same effective act in the analysis of qualitative properties of solutions of dynamic equations [2, 3, 9]. To the best of the author's knowledge there are few inequalities involving higher order derivatives 
established in the literature [10-13]. In the following, we recall some of these results that serve and motivate the contents of this paper.

In [13] the authors proved that if $y:[a, b]_{\mathbb{T}} \rightarrow \mathbb{R}$ is delta differentiable $n$ times with $y^{\Delta_{i}}(a)=0$, for $i=0,1, \ldots, n-1$, and $h$ is a positive rd-continuous function on $[a, b]_{\mathbb{T}}$, then

$$
\int_{a}^{b} h(t)|y(t)|^{p}\left|y^{\Delta_{n}}(t)\right|^{q} \Delta t \leq\left(\frac{q}{p+q}\right)(b-a)^{n p} \int_{a}^{b} h(t)\left|y^{\Delta_{n}}(t)\right|^{p+q} \Delta t
$$

In [10] it is proved that if $y:[a, b]_{\mathbb{T}} \rightarrow \mathbb{R}$ is delta differentiable $n$ times ( $n$ odd) with $y^{\Delta_{i}}(a)=$ 0 , for $i=0,1, \ldots, n-1$, then

$$
\int_{a}^{b}|y(t)| \Delta t \leq\left(\int_{a}^{b}\left(\int_{a}^{t} h_{n-1}^{p}(t, \sigma(s)) \Delta s\right)^{q / p} \Delta t\right) \int_{a}^{b}\left|y^{\Delta_{n}}(t)\right|^{q} \Delta t
$$

where $p, q>1$ and satisfy $1 / p+1 / q=1$. Also in [10] it is proved that if $y:[a, b]_{\mathbb{T}} \rightarrow \mathbb{R}$ is delta differentiable $n$ times with $y^{\Delta_{i}}(a)=0$, for $i=0,1, \ldots, n-1$, and $\left|y^{\Delta_{n}}(t)\right|$ is increasing, then

$$
\int_{a}^{b}|y(t)|\left|y^{\Delta_{n}}(t)\right| \Delta t \leq(b-a)^{1 / q}\left(\int_{a}^{b}\left(\int_{a}^{t} h_{n-1}^{p}(t, \sigma(s)) \Delta s\right)^{1 / p} \Delta t\right)\left(\int_{a}^{b}\left|y^{\Delta_{n}}(t)\right|^{2 q} \Delta t\right)^{1 / q},
$$

where $p, q>1$ and satisfy $1 / p+1 / q=1$. As a generalization of (1.3) it is proved in [10] that if $y:[a, b]_{\mathbb{T}} \rightarrow \mathbb{R}$ is delta differentiable $n$ times with $y^{\Delta_{m+i}}(a)=0$, for $i=0,1, \ldots, n-m-1$, and $\left|y^{\Delta_{n}}(t)\right|$ is increasing, then

$$
\begin{aligned}
& \int_{a}^{b}\left|y^{\Delta_{m}}(t)\right|\left|y^{\Delta_{n}}(t)\right| \Delta t \\
& \quad \leq(b-a)^{1 / q}\left(\int_{a}^{b}\left(\int_{a}^{t} h_{n-m-1}^{p}(t, \sigma(s)) \Delta s\right)^{1 / p} \Delta t\right)\left(\int_{a}^{b}\left|y^{\Delta_{n}}(t)\right|^{2 q} \Delta t\right)^{1 / q},
\end{aligned}
$$

where $p, q>1$ and satisfy $1 / p+1 / q=1$. In [12] the authors proved that if $r$ and $s$ are positive rd-continuous functions on $[a, b]_{\mathbb{T}}$ such that $s$ is nonincreasing, and $y:[a, b]_{\mathbb{T}} \rightarrow \mathbb{R}$ is delta differentiable $n$ times with $y^{\Delta_{i}}(a)=0$, for $i=0,1, \ldots, n-1$, then

$$
\begin{aligned}
& \int_{a}^{b} s(x)|y(t)|^{p}\left|y^{\Delta_{n}}(t)\right| \Delta t \\
& \quad \leq \frac{1}{p+1}(b-a)^{n-1}\left(\int_{a}^{b} r^{1-\gamma}(t) \Delta t\right)^{(1+p) / \gamma}\left(\int_{a}^{b} r(t)(s(t))^{p /(p+1)}\left|y^{\Delta_{n}}(t)\right|^{v} \Delta t\right)^{(1+p) / v},
\end{aligned}
$$

where $p>0$ and $1 / \gamma+1 / v=1$. For contributions of different types of dynamic inequalities on time scales, we refer the reader to the papers $[1,2,14-17]$ and the references cited therein. 
Following this trend, to develop the qualitative theory of dynamic inequalities on time scales, we will prove some new inequalities of Opial's type involving higher order derivatives by making use of the Hölder inequality (see, [18, Theorem 6.13]):

$$
\int_{a}^{h}|f(t) g(t)| \Delta t \leq\left[\int_{a}^{h}|f(t)|^{\gamma} \Delta t\right]^{1 / \gamma}\left[\int_{a}^{h}|g(t)|^{v} \Delta t\right]^{1 / v},
$$

where $a, h \in \mathbb{T}$ and $f ; g \in C_{\mathrm{rd}}(\mathbb{I}, \mathbb{R}), \gamma>1$ and $1 / v+1 / \gamma=1$, the formula

$$
\left(x^{\gamma}(t)\right)^{\Delta}=\gamma \int_{0}^{1}\left[h x^{\sigma}+(1-h) x\right]^{\gamma-1} d h x^{\Delta}(t)
$$

which is a simple consequence of Keller's chain rule [18, Theorem 1.90], and the Taylor monomials on time scales. The results in this paper extend and improve the pervious results in the sense that our results contain two different weighted functions and do not require the monotonicity condition on $\left|y^{\Delta_{n}}(t)\right|$ (the results in [10] required that $\left|y^{\Delta_{n}}(t)\right|$ should be increasing). Some results on continuous and discrete spaces, which lead to differential and difference inequalities, will be derived from our results as special cases. This paper is a continuation of the papers $[3,10-13,16]$.

\section{Main Results}

In this section, we will prove the main results. For completeness, we recall the following concepts related to the notion of time scales. A time scale $\mathbb{T}$ is an arbitrary nonempty closed subset of the real numbers $\mathbb{R}$. We assume throughout that $\mathbb{T}$ has the topology that it inherits from the standard topology on the real numbers $\mathbb{R}$. The forward jump operator and the backward jump operator are defined by

$$
\sigma(t):=\inf \{s \in \mathbb{T}: s>t\}, \quad \rho(t):=\sup \{s \in \mathbb{T}: s<t\},
$$

where $\sup \emptyset=\inf \mathbb{T}$. A point $t \in \mathbb{T}$ is said to be left-dense if $\rho(t)=t$ and $t>\inf \mathbb{T}$, is rightdense if $\sigma(t)=t$, is left-scattered if $\rho(t)<t$ and right-scattered if $\sigma(t)>t$. The three most popular examples of calculus on time scales are differential calculus, difference calculus, and quantum calculus (see [4]), that is, when $\mathbb{T}=\mathbb{R}, \mathbb{T}=\mathbb{N}$ and $\mathbb{T}=q^{\mathbb{N}_{0}}=\left\{q^{t}: t \in \mathbb{N}_{0}\right\}$ where $q>1$. For more details of time scale analysis we refer the reader to the two books by Bohner and Peterson $[18,19]$ which summarize and organize much of the time scale calculus.

A function $g: \mathbb{T} \rightarrow \mathbb{R}$ is said to be right-dense continuous (rd-continuous) provided $g$ is continuous at right-dense points and at left-dense points in $\mathbb{T}$; left-hand limits exist and are finite. The set of all such rd-continuous functions is denoted by $C_{\mathrm{rd}}(\mathbb{T})$.

The graininess function $\mu$ for a time scale $\mathbb{T}$ is defined by $\mu(t):=\sigma(t)-t$, and for any function $f: \mathbb{T} \rightarrow \mathbb{R}$ the notation $f^{\sigma}(t)$ denotes $f(\sigma(t))$. We will assume that $\sup \mathbb{T}=\infty$ and define the time scale interval $[a, b]_{\mathbb{T}}$ by $[a, b]_{\mathbb{T}}:=[a, b] \cap \mathbb{T}$. Fix $t \in \mathbb{T}$ and let $y: \mathbb{T} \rightarrow \mathbb{R}$. 
Define $y^{\Delta}(t)$ to be the number (if it exists) with the property that given any $\epsilon>0$ there is a neighborhood $U$ of $t$ with

$$
\left|[y(\sigma(t))-y(s)]-y^{\Delta}(t)[\sigma(t)-s]\right| \leq \epsilon|\sigma(t)-s|, \quad \forall s \in U
$$

In this case, we say $y^{\Delta}(t)$ is the (delta) derivative of $y$ at $t$ and that $y$ is (delta) differentiable at $t$. We will frequently use the following results which are due to Hilger [20]. Assume that $y: \mathbb{T} \rightarrow \mathbb{R}$ and let $t \in \mathbb{T}$.

(i) If $y$ is differentiable at $t$, then $y$ is continuous at $t$.

(ii) If $y$ is continuous at $t$ and $t$ is right-scattered, then $y$ is differentiable at $t$ with

$$
y^{\Delta}(t)=\frac{y(\sigma(t))-y(t)}{\mu(t)} .
$$

(iii) If $y$ is differentiable and $t$ is right-dense, then

$$
y^{\Delta}(t)=\lim _{s \rightarrow t} \frac{y(t)-y(s)}{t-s} .
$$

(iv) If $y$ is differentiable at $t$, then $y(\sigma(t))=y(t)+\mu(t) y^{\Delta}(t)$.

We will make use of the following product and quotient rules for the derivative of the product $f g$ and the quotient $f / g$ (where $g g^{\sigma} \neq 0$, here $g^{\sigma}=g \circ \sigma$ ) of two differentiable functions $f$ and $g$ :

$$
(f g)^{\Delta}=f^{\Delta} g+f^{\sigma} g^{\Delta}=f g^{\Delta}+f^{\Delta} g^{\sigma}, \quad\left(\frac{f}{g}\right)^{\Delta}=\frac{f^{\Delta} g-f g^{\Delta}}{g g^{\sigma}} .
$$

In this paper, we will refer to the (delta) integral which we can define as follows: if $G^{\Delta}(t)=$ $g(t)$, then the Cauchy (delta) integral of $g$ is defined by

$$
\int_{a}^{t} g(s) \Delta s:=G(t)-G(a)
$$

It can be shown (see [18]) that if $g \in C_{\mathrm{rd}}(\mathbb{T})$, then the Cauchy integral $G(t):=\int_{t_{0}}^{t} g(s) \Delta s$ exists, $t_{0} \in \mathbb{T}$, and satisfies $G^{\Delta}(t)=g(t), t \in \mathbb{T}$. An infinite integral is defined as

$$
\int_{a}^{\infty} f(t) \Delta t=\lim _{b \rightarrow \infty} \int_{a}^{b} f(t) \Delta t
$$

and the integration on discrete time scales is defined by

$$
\int_{a}^{b} f(t) \Delta t=\sum_{t \in[a, b)} \mu(t) f(t)
$$


Now, we define the Taylor monomials or generalized polynomials as defined originally by Agarwal and Bohner [21]. These types of monomials are important because they are intimately related to Cauchy functions for certain dynamic equations which are important in variations of constants formulas. The Taylor monomials $h_{k}: \mathbb{T} \times \mathbb{T} \rightarrow \mathbb{R}, k \in \mathbb{N}_{0}=\mathbb{N} \cup\{0\}$ are defined recursively as follows. The function $h_{0}$ is defined by

$$
h_{0}(t, s)=1, \quad \forall s, t \in \mathbb{T},
$$

and given $h_{k}$ for $k \in \mathbb{N}_{0}$, the function $h_{k+1}$ is defined by

$$
h_{k+1}(t, s)=\int_{s}^{t} h_{k}(\tau, s) \Delta \tau, \quad \forall s, t \in \mathbb{T}
$$

If we let $h_{k}^{\Delta}(t, s)$ denote for each fixed $s \in \mathbb{T}$, the derivative of $h(t, s)$ with respect to $t$, then

$$
h_{k}^{\Delta}(t, s)=h_{k-1}(t, s), \quad k \in \mathbb{N}, t \in \mathbb{T},
$$

for each fixed $s \in \mathbb{T}$. The above definition obviously implies

$$
h_{1}(t, s)=t-s, \quad \forall s, t \in \mathbb{T} \text {. }
$$

In the following, we give some formulas of $h_{k}(t, s)$ as determined in [18]. In the case when $\mathbb{T}=\mathbb{R}$, then $\sigma(t)=t, \mu(t)=0, y^{\Delta}(t)=y^{\prime}(t)$, and

$$
h_{k}(t, s)=\frac{(t-s)^{k}}{k !}, \quad \forall s, t \in \mathbb{R}
$$

In the case when $\mathbb{T}=\mathbb{N}$, we see that $\sigma(t)=t+1, \mu(t)=1, y^{\Delta}(t)=\Delta y(t)=y(t+1)-y(t)$, and

$$
h_{k}(n, s):=\frac{(n-s)^{(k)}}{k !}, \quad k=0,1,2, \ldots, t>s,
$$

where $t^{(k)}=t(t-1) \cdots(t-k+1)$ is the so-called falling function (cf. [22]). When $\mathbb{T}=\{t: t=$ $\left.q^{n}, n \in \mathbb{N}, q>1\right\}$, we have $\sigma(t)=q t, \mu(t)=(q-1) t, y^{\Delta}(t)=\Delta_{q} y(t)=(y(q t)-y(t)) /(q-1) t$, and

$$
h_{k}(t, s)=\prod_{m=0}^{k-1} \frac{t-q^{m} s}{\sum_{j=0}^{m} q^{j}}, \quad \forall s, t \in \mathbb{T}
$$

If $\mathbb{T}=h \mathbb{N}, h>0$, we see that $\sigma(t)=t+h, \mu(t)=h, y^{\Delta}(t)=\Delta_{h} y(t)=(y(t+h)-y(t)) / h$, and

$$
h_{k}(t, s)=\frac{\prod_{i=0}^{k-1}(t-i h-s)}{k !}, \quad \forall s, t \in \mathbb{T}, t>s
$$


In general for $t \geq s$, we have that $h_{k}(t, s) \geq 0$, and

$$
h_{k}(t, s) \leq \frac{(t-s)^{k}}{k !}, \quad \forall t>s, \quad k \in \mathbb{N}_{0}
$$

We also consider the Taylor monomials $g_{k}: \mathbb{T} \times \mathbb{T} \rightarrow \mathbb{R}, k \in \mathbb{N}_{0}=\mathbb{N} \cup\{0\}$, which are defined recursively as follows. The function $g_{0}$ is defined by

$$
g_{0}(t, s)=1, \quad \forall s, t \in \mathbb{T},
$$

and given $g_{k}$ for $k \in \mathbb{N}_{0}$, the function $g_{k+1}$ is defined by

$$
g_{k+1}(t, s)=\int_{s}^{t} g_{k}(\sigma(\tau), s) \Delta \tau, \quad \forall s, t \in \mathbb{T}
$$

If we let $g_{k}^{\Delta}(t, s)$ denote for each fixed $s \in \mathbb{T}$, the derivative of $g(t, s)$ with respect to $t$, then

$$
g_{k}^{\Delta}(t, s)=g_{k-1}(\sigma(t), s), \quad k \in \mathbb{N}, t \in \mathbb{T},
$$

for each fixed $s \in \mathbb{T}$. By Theorem 1.112 in [18], we see that

$$
h_{k}(t, s)=(-1)^{k} g_{k}(s, t)
$$

We denote by $C_{\mathrm{rd}}^{(n)}(\mathbb{T})$ the space of all functions $f \in C_{\mathrm{rd}}(\mathbb{T})$ such that $f^{\Delta_{i}} \in C_{\mathrm{rd}}(\mathbb{T})$ for $i=$ $0,1,2, \ldots, n$ for $n \in \mathbb{N}$. For the function $f: \mathbb{T} \rightarrow \mathbb{R}$, we consider the second derivative $f^{\Delta_{2}}$ provided $f^{\Delta}$ is delta differentiable on $\mathbb{T}$ with derivative $f^{\Delta_{2}}=\left(f^{\Delta}\right)^{\Delta}$. Similarly, we define the $n$th order derivative $f^{\Delta_{n}}=\left(f^{\Delta_{n-1}}\right)^{\Delta}$. Now, we are ready to state the Taylor formula that we will need to prove the main results in this paper. This formula as proved in [23] states the following. Assuming that $f \in C_{\mathrm{rd}}^{(n)}(\mathbb{T})$ and $s \in \mathbb{T}$, then

$$
f(t)=\sum_{k=0}^{n-1} f^{\Delta_{k}}(s) h_{k}(t, s)+\int_{s}^{t} h_{n-1}(t, \sigma(\tau)) f^{\Delta_{n}}(\tau) \Delta \tau .
$$

As a special case if $m<n$, then

$$
f^{\Delta_{m}}(t)=\sum_{k=0}^{n-m-1} f^{\Delta_{k+m}}(s) h_{k}(t, s)+\int_{s}^{t} h_{n-m-1}(t, \sigma(\tau)) f^{\Delta_{n}}(\tau) \Delta \tau
$$

Now, we are ready to state and prove our main results in this paper. Throughout the rest of the paper, we will assume that all the integrals that will appear in the inequalities exist and are finite. 
Theorem 2.1. Letting $\mathbb{T}$ be a time scale with $a, b \in \mathbb{T}$ and $y \in C_{\mathrm{rd}}^{(n)}([a, b] \cap \mathbb{T})$. If $y^{\Delta_{i}}(a)=0$, for $i=0,1, \ldots, n-1$, then

$$
\int_{a}^{b}|y(t)|\left|y^{\Delta_{n}}(t)\right| \Delta t \leq \sqrt{\frac{1}{2}}\left(\int_{a}^{b}\left(\int_{a}^{t}\left|h_{n-1}(t, \sigma(s))\right|^{2} \Delta s\right) \Delta t\right)^{1 / 2} \int_{a}^{b}\left|y^{\Delta_{n}}(t)\right|^{2} \Delta t .
$$

Proof. From the Taylor formula (2.22), since $y^{\Delta_{i}}(a)=0$, for $i=0,1, \ldots, n-1$, we have

$$
y(t):=\int_{a}^{t} h_{n-1}(t, \sigma(s)) y^{\Delta_{n}}(s) \Delta s
$$

This implies that

$$
\left|y^{\Delta_{n}}(t)\right||y(t)| \leq\left|y^{\Delta_{n}}(t)\right| \int_{a}^{t}\left|h_{n-1}(t, \sigma(s))\right|\left|y^{\Delta_{n}}(s)\right| \Delta s .
$$

Applying the Hölder inequality (1.6) with $\gamma=v=2$, we have

$$
\left|y^{\Delta_{n}}(t)\right||y(t)| \leq\left|y^{\Delta_{n}}(t)\right|\left(\int_{a}^{t}\left|h_{n-1}(t, \sigma(s))\right|^{2} \Delta s\right)^{1 / 2}\left(\int_{a}^{t}\left|y^{\Delta_{n}}(s)\right|^{2} \Delta s\right)^{1 / 2} .
$$

Then

$$
\int_{a}^{b}\left|y^{\Delta_{n}}(t)\right||y(t)| \Delta t \leq \int_{a}^{b}\left(\int_{a}^{t}\left|h_{n-1}(t, \sigma(s))\right|^{2} \Delta s\right)^{1 / 2}\left|y^{\Delta_{n}}(t)\right|\left(\int_{a}^{t}\left|y^{\Delta_{n}}(s)\right|^{2} \Delta s\right)^{1 / 2} \Delta t .
$$

Define $z(t):=\int_{a}^{t}\left|y^{\Delta_{n}}(s)\right|^{2} \Delta s$. This implies that $z(a)=0$ and $\left|y^{\Delta_{n}}(t)\right|^{2}=z^{\Delta}(t)$. From this and (2.28), we have

$$
\int_{a}^{b}\left|y^{\Delta_{n}}(t)\right||y(t)| \Delta t \leq \int_{a}^{b}\left(\int_{a}^{t}\left|h_{n-1}(t, \sigma(s))\right|^{2} \Delta s\right)^{1 / 2}\left(z(t) z^{\Delta}(t)\right)^{1 / 2} \Delta t .
$$

Applying the Hölder inequality again $\gamma=v=2$, we obtain

$$
\int_{a}^{b}\left|y^{\Delta_{n}}(t)\right||y(t)| \Delta t \leq\left(\int_{a}^{b}\left(\int_{a}^{t}\left|h_{n-1}(t, \sigma(s))\right|^{2} \Delta s\right) \Delta t\right)^{1 / 2}\left(\int_{a}^{b} z(t) z^{\Delta}(t) \Delta t\right)^{1 / 2} .
$$

From (1.7), we have (note that $z(t)>0$ and $z^{\Delta}(t)>0$ ) that

$$
\left(z^{2}(t)\right)^{\Delta}=2 z^{\Delta}(t) \int_{0}^{1}\left[h z^{\sigma}+(1-h) z\right] d h \geq 2 z^{\Delta}(t) z(t) .
$$


Then

$$
\int_{a}^{b} z(t) z^{\Delta}(t) \Delta t \leq \frac{1}{2} \int_{a}^{b}\left(z^{2}(t)\right)^{\Delta} \Delta t=\frac{1}{2} z^{2}(b),
$$

where $z(a)=0$. Substituting (2.32) into (2.30), we have

$$
\begin{aligned}
\int_{a}^{b}\left|y^{\Delta_{n}}(t)\right||y(t)| \Delta t & \leq \sqrt{\frac{1}{2}}\left(\int_{a}^{b}\left(\int_{a}^{t}\left|h_{n-1}(t, \sigma(s))\right|^{2} \Delta s\right) \Delta t\right)^{1 / 2} z(b) \\
& =\sqrt{\frac{1}{2}}\left(\int_{a}^{b}\left(\int_{a}^{t}\left|h_{n-1}(t, \sigma(s))\right|^{2} \Delta s\right) \Delta t\right)^{1 / 2} \int_{a}^{b}\left|y^{\Delta_{n}}(t)\right|^{2} \Delta t
\end{aligned}
$$

which is the desired inequality (2.24). The proof is complete.

Remark 2.2. Let $0 \leq k<n$, but fixed, and let $x(t) \in C_{\mathrm{rd}}^{(n-k)}([a, b] \cap \mathbb{T})$ be such that $x^{\left(\Delta_{i}\right)}(a)=0$, $0 \leq i \leq n-k-1$. Then from (2.24) it follows that

$$
\int_{a}^{b}|x(t)|\left|x^{\Delta_{n-k}}(t)\right| \Delta t \leq \sqrt{\frac{1}{2}}\left(\int_{a}^{b}\left(\int_{a}^{t}\left|h_{n-k-1}(t, \sigma(s))\right|^{2} \Delta s\right) \Delta t\right)^{1 / 2} \int_{a}^{b}\left|x^{\Delta_{n-k}}(t)\right|^{2} \Delta t .
$$

Thus for $x=y^{\Delta_{k}}$, where $y \in C_{\mathrm{rd}}^{(n-k)}[a, b], y^{\left(\Delta_{i}\right)}=0, k \leq i \leq n-1$; then we have the following result.

Corollary 2.3. Let $\mathbb{T}$ be a time scale with $a, b \in \mathbb{T}$ and $y \in C_{\mathrm{rd}}^{(n)}([a, b] \cap \mathbb{T})$. If $y^{\left(\Delta_{i}\right)}(a)=0$, $k \leq i \leq n-1$, then

$$
\int_{a}^{b}\left|y^{\Delta_{k}}(t)\right|\left|y^{\Delta_{n}}(t)\right| \Delta t \leq \sqrt{\frac{1}{2}}\left(\int_{a}^{b}\left(\int_{a}^{t}\left|h_{n-k-1}(t, \sigma(s))\right|^{2} \Delta s\right) \Delta t\right)^{1 / 2} \int_{a}^{b}\left|y^{\Delta_{n}}(t)\right|^{2} \Delta t .
$$

Note that Theorem 2.1 can be extended to a general inequality with two different constants $p$ and $q$ that satisfy $1 / p+1 / q=1$, to obtain the following result.

Theorem 2.4. Letting $\mathbb{T}$ be a time scale with $a, b \in \mathbb{T}$ and $y \in C_{\mathrm{rd}}^{(n)}([a, b] \cap \mathbb{T})$. If $y^{\Delta_{i}}(a)=0$, for $i=0,1, \ldots, n-1$, then

$$
\int_{a}^{b}|y(t)|\left|y^{\Delta_{n}}(t)\right| \Delta t \leq\left(\frac{1}{2}\right)^{1 / q}\left(\int_{a}^{b}\left(\int_{a}^{t}\left|h_{n-1}(t, \sigma(s))\right|^{p} \Delta s\right) \Delta t\right)^{1 / p} \int_{a}^{b}\left|y^{\Delta_{n}}(t)\right|^{q} \Delta t .
$$


Theorem 2.5. Let $\mathbb{T}$ be a time scale with $a, b \in \mathbb{T}$ and let $l, m$ be positive real numbers such that $l+m>1$, and $y \in C_{\mathrm{rd}}^{(n)}([a, b] \cap \mathbb{T})$. If $y^{\Delta_{i}}(a)=0$, for $i=0,1, \ldots, n-1$; then

$$
\int_{a}^{b}|y(t)|^{l}\left|y^{\Delta_{n}}(t)\right|^{m} \Delta t \leq\left(\frac{m}{l+m}\right)^{m /(l+m)}\left(\int_{a}^{b} H^{(l+m-1)}(t, s) \Delta t\right)^{l /(l+m)} \int_{a}^{b}\left|y^{\Delta_{n}}(t)\right|^{l+m} \Delta t
$$

where

$$
H(t, s):=\int_{a}^{t}\left(h_{n-1}(t, \sigma(s))\right)^{(l+m) /(l+m-1)} \Delta s
$$

Proof. From the Taylor formula (2.22) and since $y^{\Delta_{i}}(a)=0$, for $i=0,1, \ldots, n-1$, we have

$$
|y(t)| \leq \int_{a}^{t} h_{n-1}(t, \sigma(s))\left|y^{\Delta_{n}}(s)\right| \Delta s
$$

Applying the Hölder inequality with $\gamma=l+m$ and $v=(l+m) /(l+m-1)$, we have

$$
|y(t)| \leq\left(\int_{a}^{t}\left(h_{n-1}(t, \sigma(s))\right)^{(l+m) /(l+m-1)} \Delta s\right)^{(l+m-1) /(l+m)}\left(\int_{a}^{t}\left|y^{\Delta_{n}}(s)\right|^{l+m} \Delta s\right)^{1 /(l+m)} .
$$

This implies that

$$
\begin{aligned}
& |y(t)|^{l}\left|y^{\Delta_{n}}(t)\right|^{m} \\
& \quad \leq\left|y^{\Delta_{n}}(t)\right|^{m}\left(\int_{a}^{t}\left(h_{n-1}(t, \sigma(s))\right)^{(l+m) /(l+m-1)} \Delta s\right)^{l((l+m-1) /(l+m))}\left(\int_{a}^{t}\left|y^{\Delta_{n}}(s)\right|^{l+m} \Delta s\right)^{l /(l+m)} .
\end{aligned}
$$

Then

$$
\begin{aligned}
& \int_{a}^{b}\left|y^{\Delta_{n}}(t)\right|^{m}|y(t)|^{l} \Delta t \\
& \quad \leq \int_{a}^{b}\left(\int_{a}^{t}\left(h_{n-1}(t, \sigma(s))\right)^{(l+m) /(l+m-1)} \Delta s\right)^{l((l+m-1) /(l+m))}\left|y^{\Delta_{n}}(t)\right|^{m}\left(\int_{a}^{t}\left|y^{\Delta_{n}}(s)\right|^{l+m} \Delta s\right)^{l /(l+m)} \Delta t .
\end{aligned}
$$

Define $z(t):=\int_{a}^{t}\left|y^{\Delta_{n}}(s)\right|^{l+m} \Delta s$. This implies that $z(a)=0$, and

$$
\left|y^{\Delta_{n}}(t)\right|^{m}=\left(z^{\Delta}(t)\right)^{m /(l+m)}>0
$$


From this and (2.42), we have

$$
\begin{aligned}
& \int_{a}^{b}|y(t)|^{l}\left|y^{\Delta_{n}}(t)\right|^{m} \Delta t \\
& \quad \leq \int_{a}^{b}\left(\int_{a}^{t}\left(h_{n-1}(t, \sigma(s))\right)^{(l+m) /(l+m-1)} \Delta s\right)^{l((l+m-1) /(l+m))}\left(z^{\Delta}(t)\right)^{m /(l+m)}(z(t))^{l /(l+m)} \Delta t .
\end{aligned}
$$

Applying the Hölder inequality again $\gamma=(l+m) / l$ and $v=(l+m) / m$, we have

$$
\begin{aligned}
& \int_{a}^{b}|y(t)|^{l}\left|y^{\Delta_{n}}(t)\right|^{m} \Delta t \\
& \quad \leq\left(\int_{a}^{b}\left(\int_{a}^{t}\left(h_{n-1}(t, \sigma(s))\right)^{(l+m) /(l+m-1)} \Delta s\right)^{l((l+m-1) /(l+m))((l+m) / l)} \Delta t\right)^{l /(l+m)} \\
& \quad \times\left(\int_{a}^{b} z^{\Delta}(t)(z(t))^{(l / m)} \Delta t\right)^{m /(m+l)} .
\end{aligned}
$$

From (1.7), we have (note that $z(t)$ and $z^{\Delta}(t)>0$; see also, page 116 [17]) that

$$
\begin{aligned}
\left(z^{(l+m) / m}(t)\right)^{\Delta} & \geq \frac{l+m}{m} \int_{0}^{1}\left[h z^{\sigma}+(1-h) z\right]^{((l+m) / m)-1} z^{\Delta}(t) \\
& \geq \frac{l+m}{m}(z(t))^{(l / m)} z^{\Delta}(t) .
\end{aligned}
$$

Then

$$
\int_{a}^{b} z^{(l / m)}(t) z^{\Delta}(t) \Delta t \leq \frac{m}{l+m} \int_{a}^{b}\left(z^{(l+m) / m}(t)\right)^{\Delta} \Delta t=\frac{m}{l+m} z^{(l+m) / m}(b),
$$


where $z(a)=0$. Substituting (2.47) into (2.45), we have

$$
\begin{aligned}
& \int_{a}^{b}|y(t)|^{l}\left|y^{\Delta_{n}}(t)\right|^{m} \Delta t \\
& \leq\left(\frac{m}{l+m}\right)^{m /(l+m)}\left(\int_{a}^{b}\left(\int_{a}^{t}\left(h_{n-1}(t, \sigma(s))\right)^{(l+m) /(l+m-1)} \Delta s\right)^{l((l+m-1) /(l+m))((l+m) / l)} \Delta t\right)^{l /(l+m)} z(b) \\
& =\left(\frac{m}{l+m}\right)^{m /(l+m)}\left(\int_{a}^{b}\left(\int_{a}^{t}\left(h_{n-1}(t, \sigma(s))\right)^{(l+m) /(l+m-1)} \Delta s\right)^{(l+m-1)} \Delta t\right)^{l /(l+m)} \\
& \quad \times \int_{a}^{b}\left|y^{\Delta_{n}}(t)\right|^{l+m} \Delta t
\end{aligned}
$$

which is the desired inequality (2.37). The proof is complete.

Following Remark 2.2, we can obtain the following result.

Corollary 2.6. Let $\mathbb{T}$ be a time scale with $a, b \in \mathbb{T}$ and let $l, m$ be positive real numbers such that $l+m>1$ and $y \in C_{\mathrm{rd}}^{(n)}([a, b] \cap \mathbb{T})$. If $y^{\left(\Delta_{i}\right)}(a)=0, k \leq i \leq n-1 ;$ then

$$
\int_{a}^{b}\left|y^{\Delta_{k}}(t)\right|^{l}\left|y^{\Delta_{n}}(t)\right|^{m} \Delta t \leq\left(\frac{m}{l+m}\right)^{m /(l+m)}\left(\int_{a}^{b} H^{(l+m-1)}(t, s) \Delta t\right)^{l /(l+m)} \int_{a}^{b}\left|y^{\Delta_{n}}(t)\right|^{l+m} \Delta t,
$$

where

$$
H(t, s):=\int_{a}^{t}\left(h_{n-k-1}(t, \sigma(s))\right)^{(l+m) /(l+m-1)} \Delta s .
$$

Note that Theorem 2.5 cannot be applied when $l+m=1$. In the following theorem we prove a new inequality which can be applied in this case.

Theorem 2.7. Let $\mathbb{T}$ be a time scale with $a, b \in \mathbb{T}$ and let $l, m$ be positive real numbers such that $l+m=1$ and $y \in C_{\mathrm{rd}}^{(n)}([a, b] \cap \mathbb{T})$. If $y^{\Delta_{i}}(a)=0$, for $i=0,1, \ldots, n-1$, then

$$
\int_{a}^{b}|y(t)|^{l}\left|y^{\Delta_{n}}(t)\right|^{m} \Delta t \leq m^{m}\left(\int_{a}^{b} h_{n-1}(t, a) \Delta t\right) \int_{a}^{b}\left|y^{\Delta_{n}}(t)\right| \Delta t
$$


Proof. Using the fact that $\left|h_{n}(t, s)\right|$ is increasing with respect to its first component for $t \geq$ $\sigma(s)>a$, we have from the Taylor formula (2.22) and $y^{\Delta_{i}}(a)=0$, for $i=0,1, \ldots, n-1$, that

$$
|y(t)| \leq h_{n-1}(t, a) \int_{a}^{t}\left|y^{\Delta_{n}}(s)\right| \Delta s
$$

This implies that

$$
|y(t)|^{l}\left|y^{\Delta_{n}}(t)\right|^{m} \leq\left(h_{n-1}(t, a)\right)^{l}\left|y^{\Delta_{n}}(t)\right|^{m}\left(\int_{a}^{t}\left|y^{\Delta_{n}}(s)\right| \Delta s\right)^{l} .
$$

Now applying the Hölder inequality (1.6) with $\gamma=1 / l$ and $v=1 / m$, we obtain

$$
\int_{a}^{b}|y(t)|^{l}\left|y^{\Delta_{n}}(t)\right|^{m} \Delta t \leq\left(\int_{a}^{b} h_{n-1}(t, a) \Delta t\right)^{l}\left(\int_{a}^{b}\left|y^{\Delta_{n}}(t)\right|\left(\int_{a}^{t}\left|y^{\Delta_{n}}(s)\right| \Delta s\right)^{l / m} \Delta t\right)^{m} .
$$

Let $z(t)=\int_{a}^{t}\left|y^{\Delta_{n}}(s)\right| \Delta s$. Then $z(a)=0$ and $z^{\Delta}(t)=\left|y^{\Delta_{n}}(t)\right|$ so that

$$
\int_{a}^{b}\left|y^{\Delta_{n}}(t)\right|\left(\int_{a}^{t}\left|y^{\Delta_{n}}(s)\right| \Delta s\right)^{l / m} \Delta t=\int_{a}^{b} z^{\Delta}(t)(z(t))^{l / m} \Delta t
$$

and hence

$$
\int_{a}^{b}|y(t)|^{l}\left|y^{\Delta_{n}}(t)\right|^{m} \Delta t \leq\left(\int_{a}^{b} h_{n-1}(t, a) \Delta t\right)^{l}\left(\int_{a}^{b} z^{\Delta}(t)(z(t))^{l / m} \Delta t\right)^{m} .
$$

As in the proof of Theorem 2.5, we have that

$$
\begin{aligned}
\int_{a}^{b} z^{\Delta}(t)(z(t))^{l / m} \Delta t & \leq \int_{a}^{b} z^{l / m}(t) z^{\Delta}(t) \Delta t \leq m \int_{a}^{b}\left(z^{(l+m) / m}(t)\right)^{\Delta} \Delta t \\
& =m z^{1 / m}(b)=m\left(\int_{a}^{b}\left|y^{\Delta_{n}}(t)\right| \Delta t\right)^{1 / m}
\end{aligned}
$$

Substituting into (2.56), we have

$$
\int_{a}^{b}|y(t)|^{l}\left|y^{\Delta_{n}}(t)\right|^{m} \Delta t \leq m^{m}\left(\int_{a}^{b} h_{n-1}(t, a) \Delta t\right)\left(\int_{a}^{b}\left|y^{\Delta_{n}}(t)\right| \Delta t\right)
$$

which is the desired inequality (2.51). The proof is complete.

Following Remark 2.2, we can obtain the following result. 
Corollary 2.8. Let $\mathbb{T}$ be a time scale with $a, b \in \mathbb{T}$ and let $l, m$ be positive real numbers such that $l+m=1$, and $y \in C_{\mathrm{rd}}^{(n)}([a, b] \cap \mathbb{T})$. If $y^{\left(\Delta_{i}\right)}(a)=0, k \leq i \leq n-1$, then

$$
\int_{a}^{b}\left|y^{\Delta_{k}}(t)\right|^{l}\left|y^{\Delta_{n}}(t)\right|^{m} \Delta t \leq m^{m}\left(\int_{a}^{b}\left|h_{n-k-1}(t, a)\right| \Delta t\right)\left(\int_{a}^{b}\left|y^{\Delta_{n}}(t)\right| \Delta t\right) .
$$
tions.

In the following, we will prove some inequalities with two different weighted func-

Theorem 2.9. Let $\mathbb{T}$ be a time scale with $a, b \in \mathbb{T}$ and let $l, m, r$ be positive real numbers such that $l+m>1$ and $r>1$. Further, let $p(t)$ and $q(t)$ be positive rd-continuous functions defined on $[a, b] \cap \mathbb{T}$ and $y \in C_{\mathrm{rd}}^{(n)}([a, b] \cap \mathbb{T})$. If $y^{\Delta_{i}}(a)=0$, for $i=0,1, \ldots, n-1$, then

$$
\int_{a}^{b} q(t)|y(t)|^{l}\left|y^{\Delta_{n}}(t)\right|^{m} \Delta t \leq \Lambda_{1}(l, m, r, p, q)\left(\int_{a}^{b} p(s)\left|y^{\Delta_{n}}(s)\right|^{r} \Delta s\right)^{(l+m) / r}
$$

where

$$
\begin{gathered}
\Lambda_{1}(l, m, p, q, r):=\left(\frac{m}{l+m}\right)^{m / r}\left(\int_{a}^{b} q^{r /(r-m)}(t) p^{-m /(r-m)}(t)(P(t))^{l((r-1) /(r-m))} \Delta t\right)^{(r-m) / r}, \\
P(t):=\int_{a}^{t} p^{-1 /(r-1)}(s)\left(h_{n-1}(t, \sigma(s))\right)^{r /(r-1)} \Delta s .
\end{gathered}
$$

Proof. From the Taylor formula (2.22), we see that

$$
|y(t)| \leq \int_{a}^{t} p^{-1 / r}(s)\left(h_{n-1}(t, \sigma(s))\right) p^{l / r}(s)\left|y^{\Delta_{n}}(s)\right| \Delta s
$$

Applying the Hölder inequality on the right hand side with $r$ and $r / r-1$, we have

$$
\begin{aligned}
|y(t)| & \leq \int_{a}^{t} p^{-1 / r}(s) h_{n-1}(t, \sigma(s)) p^{l / r}(s)\left|y^{\Delta_{n}}(s)\right| \Delta s \\
& \leq\left(\int_{a}^{t} p^{-1 /(r-1)}(s)\left(h_{n-1}(t, \sigma(s))\right)^{r /(r-1)} \Delta s\right)^{(r-1) / r}\left(\int_{a}^{t} p(s)\left|y^{\Delta_{n}}(s)\right|^{r} \Delta s\right)^{1 / r} .
\end{aligned}
$$

This implies that

$$
q(t)|y(t)|^{l}\left|y^{\Delta_{n}}(t)\right|^{m} \leq q(t) P^{l((r-1) / r)}(t)\left|y^{\Delta_{n}}(t)\right|^{m}\left(\int_{a}^{t} p(s)\left|y^{\Delta_{n}}(s)\right|^{r} \Delta s\right)^{l / r}
$$


Integrating from $a$ to $b$, we have

$$
\begin{aligned}
& \int_{a}^{b} q(t)|y(t)|^{l}\left|y^{\Delta_{n}}(t)\right|^{m} \Delta t \\
& \quad \leq \int_{a}^{b} q(t) P^{l((r-1) / r)}(t)\left|y^{\Delta_{n}}(t)\right|^{m}\left(\int_{a}^{t} p(s)\left|y^{\Delta_{n}}(s)\right|^{r} \Delta s\right)^{l / r} \Delta t .
\end{aligned}
$$

Let

$$
z(t):=\int_{a}^{t} p(s)\left|y^{\Delta_{n}}(s)\right|^{r} \Delta s
$$

Then $z(a)=0, z^{\Delta}(t)=p(t)\left|y^{\Delta_{n}}(t)\right|^{r}$ and $\left|y^{\Delta_{n}}(t)\right|^{m}=\left(z^{\Delta}(t)\right)^{m / r} p^{-m / r}(t)$. This implies that

$$
\begin{aligned}
& \int_{a}^{b} q(t)|y(t)|^{l}\left|y^{\Delta_{n}}(t)\right|^{m} \Delta t \\
& \quad \leq \int_{a}^{b} q(t) P^{l((r-1) / r)}(t) p^{-m / r}(t)\left(z^{\Delta}(t)\right)^{m / r}(z(t))^{l / r} \Delta t .
\end{aligned}
$$

Applying the Hölder inequality (1.6) with indices $r / m$ and $r / r-m$, we obtain

$$
\begin{aligned}
& \int_{a}^{b} q(t) P^{l((r-1) / r)}(t) p^{-m / r}(t)\left(z^{\Delta}(t)\right)^{m / r}(z(t))^{l / r} \Delta t \\
& \quad \leq\left(\int_{a}^{b}\left(z^{\Delta}(t)\right)(z(t))^{l / m} \Delta t\right)^{m / r}\left(\int_{a}^{b} q^{r /(r-m)}(t) P^{l((r-1) /(r-m))}(t) p^{-m /(r-m)}(t) \Delta t\right)^{(r-m) / r} .
\end{aligned}
$$

Substituting into (2.67), we have

$$
\begin{aligned}
& \int_{a}^{b} q(t)|y(t)|^{l}\left|y^{\Delta_{n}}(t)\right|^{m} \Delta t \\
& \quad \leq\left(\int_{a}^{b} q^{r /(r-m)}(t) P^{l((r-1) /(r-m))}(t) p^{-m /(r-m)}(t) \Delta t\right)^{(r-m) / r}\left(\int_{a}^{b}\left(z^{\Delta}(t)\right)(z(t))^{l / m} \Delta t\right)^{m / r} .
\end{aligned}
$$


As in the proof of Theorem 2.5, we have

$$
\begin{aligned}
& \left(\int_{a}^{b} z^{\Delta}(t)(z(t))^{l / m} \Delta t\right)^{m / r} \\
& \quad \leq\left(\frac{m}{l+m} \int_{a}^{b}\left(z^{(l+m) / m}(t)\right)^{\Delta} \Delta t\right)^{m / r} \\
& \quad=\left(\frac{m}{l+m}\right)^{m / r}(z(b))^{(l+m) / r}=\left(\frac{m}{l+m}\right)^{m / r}\left(\int_{a}^{b} p(s)\left|y^{\Delta_{n}}(s)\right|^{r} \Delta s\right)^{(l+m) / r} .
\end{aligned}
$$

This implies that

$$
\int_{a}^{b} q(t)|y(t)|^{l}\left|y^{\Delta_{n}}(t)\right|^{m} \Delta t \leq \Lambda_{1}(l, m, p, q, r)\left(\int_{a}^{b} p(s)\left|y^{\Delta_{n}}(s)\right|^{r} \Delta s\right)^{(l+m) / r},
$$

which is the desired inequality (2.60) where $\Lambda_{1}(l, m, p, q, r)$ is defined as in (2.61). The proof is complete.

Following Remark 2.2, we can obtain the following result.

Theorem 2.10. Let $\mathbb{T}$ be a time scale with $a, b \in \mathbb{T}$ and let $l, m$ be positive real numbers such that $l+m>1$ and $r>1$. Further, let $p(t)$ and $q(t)$ be positive rd-continuous functions defined on $[a, b] \cap \mathbb{T}$ and $y \in C_{\mathrm{rd}}^{(n)}([a, b] \cap \mathbb{T})$. If $y^{\left(\Delta_{i}\right)}(a)=0, k \leq i \leq n-1$, then

$$
\int_{a}^{b} q(t)\left|y^{\Delta_{k}}(t)\right|^{l}\left|y^{\Delta_{n}}(t)\right|^{m} \Delta t \leq \Lambda_{2}(l, m, r, p, q)\left(\int_{a}^{b} p(s)\left|y^{\Delta_{n}}(s)\right|^{r} \Delta s\right)^{(l+m) / r},
$$

where

$$
\begin{gathered}
\Lambda_{2}(l, m, p, q, r):=\left(\frac{m}{l+m}\right)^{m / r}\left(\int_{a}^{b} q^{r /(r-m)}(t) p^{-m /(r-m)}(t)(P(t))^{l((r-1) /(r-m))} \Delta t\right)^{(r-m) / r}, \\
P(t):=\int_{a}^{t} p^{-1 /(r-1)}(s) h_{n-k-1}^{r /(r-1)}(t, \sigma(s)) \Delta s .
\end{gathered}
$$

Note that Theorem 2.10 cannot be applied when $r=1$ and $r<m$. In the following theorem we prove an inequality which can be applied in this case. 
Theorem 2.11. Let $\mathbb{T}$ be a time scale with $a, b \in \mathbb{T}$ and let $\alpha, \beta$ be positive real numbers such that $\alpha+\beta>1$, and let $p$, $q$ be nonnegative rd-continuous functions on $(a, b)_{\mathbb{T}}$ and $y \in C_{\mathrm{rd}}^{(n)}([a, b] \cap \mathbb{T})$. If $y^{\Delta_{i}}(a)=0$, for $i=0,1, \ldots, n-1$, then

$$
\int_{a}^{b} q(t)|y(t)|^{\alpha}\left|y^{\Delta_{n}}(t)\right|^{\beta} \Delta t \leq \Lambda_{3}(a, b, \alpha, \beta) \int_{a}^{b} p(t)\left|y^{\Delta_{n}}(t)\right|^{\alpha+\beta} \Delta t
$$

where

$$
\begin{aligned}
& \Lambda_{3}(a, b, \alpha, \beta) \\
& \quad=\left(\frac{\beta}{\alpha+\beta}\right)^{\beta /(\alpha+\beta)}\left(\int_{a}^{b} \frac{q^{(\alpha+\beta) / \alpha}(t)}{p^{\beta / \alpha}(t)}\left(\int_{a}^{t} \frac{\left(h_{n-1}(t, \sigma(s))\right)^{(\alpha+\beta) /(\alpha+\beta-1)}}{p^{1 /(\alpha+\beta-1)}(s)} \Delta s\right)^{(\alpha+\beta-1)} \Delta t\right)^{\alpha /(\alpha+\beta)} .
\end{aligned}
$$

Proof. From the Taylor formula, we see that

$$
|y(t)| \leq \int_{a}^{t} h_{n-1}(t, \sigma(s))\left|y^{\Delta_{n}}(s)\right| \Delta s=\int_{a}^{t} \frac{h_{n-1}(t, \sigma(s))}{(p(s))^{1 /(\alpha+\beta)}}(p(s))^{1 /(\alpha+\beta)}\left|y^{\Delta_{n}}(s)\right| \Delta s .
$$

Now, since $p$ is nonnegative on $(a, b)_{\mathbb{T}}$, it follows from the Hölder inequality (1.6) with

$$
\begin{gathered}
f(s)=\frac{h_{n-1}(t, \sigma(s))}{(p(s))^{1 /(\alpha+\beta)}}, \quad g(s)=(p(s))^{1 /(\alpha+\beta)}\left|y^{\Delta_{n}}(s)\right| \\
r=\frac{\alpha+\beta}{\alpha+\beta-1}, \quad v=\alpha+\beta
\end{gathered}
$$

that

$$
|y(t)| \leq\left(\int_{a}^{t} \frac{\left(h_{n-1}(t, \sigma(s))\right)^{(\alpha+\beta) /(\alpha+\beta-1)}}{(p(s))^{1 /(\alpha+\beta-1)}} \Delta s\right)^{(\alpha+\beta-1) /(\alpha+\beta)}\left(\int_{a}^{t} p(s)\left|y^{\Delta_{n}}(s)\right|^{\alpha+\beta} \Delta s\right)^{1 /(\alpha+\beta)} .
$$

Then, we get that

$$
|y(t)|^{\alpha} \leq\left(\int_{a}^{t} \frac{\left(h_{n-1}(t, \sigma(s))\right)^{(\alpha+\beta) /(\alpha+\beta-1)}}{(p(s))^{1 /(\alpha+\beta-1)}} \Delta s\right)^{\alpha((\alpha+\beta-1) /(\alpha+\beta))}\left(\int_{a}^{t} p(s)\left|y^{\Delta_{n}}(s)\right|^{\alpha+\beta} \Delta s\right)^{\alpha /(\alpha+\beta)} .
$$

Setting $z(t):=\int_{a}^{t} p(s)\left|y^{\Delta_{n}}(s)\right|^{\alpha+\beta} \Delta s$, we see that $z(a)=0$, and

$$
z^{\Delta}(t)=p(t)\left|y^{\Delta_{n}}(t)\right|^{\alpha+\beta}>0 .
$$


This gives us

$$
\left|y^{\Delta_{n}}(t)\right|^{\beta}=\left(\frac{z^{\Delta}(t)}{p(t)}\right)^{\beta /(\alpha+\beta)} .
$$

Since $q$ is nonnegative on $(a, b)_{\mathbb{T}}$, we have from (2.79) and (2.81) that

$$
\begin{aligned}
& q(t)|y(t)|^{\alpha}\left|y^{\Delta_{n}}(t)\right|^{\beta} \\
& \quad \leq q(t)\left(\frac{1}{p(t)}\right)^{\beta /(\alpha+\beta)}\left(\int_{a}^{t} \frac{\left(h_{n-1}(t, \sigma(s))\right)^{(\alpha+\beta) /(\alpha+\beta-1)}}{p^{1 /(\alpha+\beta-1)}(s)} \Delta s\right)^{\alpha((\alpha+\beta-1) /(\alpha+\beta))}(z(t))^{\alpha /(\alpha+\beta)}\left(z^{\Delta}(t)\right)^{\beta /(\alpha+\beta)} .
\end{aligned}
$$

This implies that

$$
\begin{aligned}
& \int_{a}^{b} q(t)|y(t)|^{\alpha}\left|y^{\Delta_{n}}(t)\right|^{\beta} \Delta t \\
& \leq \int_{a}^{b} q(t)\left(\frac{1}{p(t)}\right)^{\beta /(\alpha+\beta)} \\
& \quad \times\left(\int_{a}^{t} \frac{\left(h_{n-1}(t, \sigma(s))\right)^{(\alpha+\beta) /(\alpha+\beta-1)}}{p^{1 /(\alpha+\beta-1)}(s)} \Delta s\right)^{\alpha((\alpha+\beta-1) /(\alpha+\beta))}(z(t))^{\alpha /(\alpha+\beta)}\left(z^{\Delta}(t)\right)^{\beta /(\alpha+\beta)} \Delta t .
\end{aligned}
$$

Applying the Hölder inequality (1.6) with indices $(\alpha+\beta) / \alpha$ and $(\alpha+\beta) / \beta$, we have

$$
\begin{aligned}
\int_{a}^{b} q(t)|y(t)|^{\alpha}\left|y^{\Delta_{n}}(t)\right|^{\beta} \Delta t \\
\leq\left(\int_{a}^{b} \frac{s^{(\alpha+\beta) / \alpha}(t)}{p^{\beta / \alpha}(t)}\left(\int_{a}^{t} \frac{\left(h_{n-1}(t, \sigma(s))\right)^{(\alpha+\beta) /(\alpha+\beta-1)}}{p^{1 /(\alpha+\beta-1)}(s)} \Delta s\right)^{(\alpha+\beta-1)} \Delta t\right)^{\alpha /(\alpha+\beta)} \\
\quad \times\left(\int_{a}^{b} z^{\alpha / \beta}(t) z^{\Delta}(t) \Delta t\right)^{\beta /(\alpha+\beta)} .
\end{aligned}
$$

From (2.80), the chain rule (1.7) and the fact that $z^{\Delta}(s)>0$, we obtain

$$
z^{\alpha / \beta}(t) z^{\Delta}(t) \leq \frac{\beta}{\alpha+\beta}\left(z^{(\alpha+\beta) / \beta}(t)\right)^{\Delta}
$$


Substituting (2.85) into (2.84) and using the fact that $z(a)=0$, we have

$$
\begin{aligned}
\int_{a}^{b} q(t)|y(t)|^{\alpha}\left|y^{\Delta_{n}}(t)\right|^{\beta} \Delta t \\
\leq\left(\int_{a}^{b} \frac{q^{(\alpha+\beta) / \alpha}(t)}{p^{\beta / \alpha}(t)}\left(\int_{a}^{t} \frac{\left(h_{n-1}(t, \sigma(s))\right)^{(\alpha+\beta) /(\alpha+\beta-1)}}{p^{1 /(\alpha+\beta-1)}(s)} \Delta s\right)^{(\alpha+\beta-1)} d t\right)^{\alpha /(\alpha+\beta)} \\
\quad \times\left(\frac{\alpha}{\alpha+\beta}\right)^{\beta /(\alpha+\beta)}\left(\int_{a}^{b}\left(z^{(\alpha+\beta) / \beta}(s)\right)^{\Delta} \Delta s\right)^{\beta /(\alpha+\beta)} \\
=\left(\int_{a}^{b} \frac{q^{(\alpha+\beta) / \alpha}(t)}{p^{\beta / \alpha}(t)}\left(\int_{a}^{t} \frac{\left(h_{n-1}(t, \sigma(s))\right)^{(\alpha+\beta) /(\alpha+\beta-1)}}{p^{1 /(\alpha+\beta-1)}(s)} \Delta s\right)^{(\alpha+\beta-1)} \Delta t\right)^{\alpha /(\alpha+\beta)} \\
\quad \times\left(\frac{\beta}{\alpha+\beta}\right)^{\beta /(\alpha+\beta)} z(b) .
\end{aligned}
$$

Using $z(b):=\int_{a}^{b} p(s)\left|y^{\Delta_{n}}(s)\right|^{\alpha+\beta} \Delta s$, we have from the last inequality that

$$
\int_{a}^{b} q(t)|y(t)|^{\alpha}\left|y^{\Delta_{n}}(t)\right|^{\beta} \Delta t \leq \Lambda_{3}(a, b, \alpha, \beta) \int_{a}^{b} p(t)\left|y^{\Delta_{n}}(t)\right|^{\alpha+\beta} \Delta t
$$

which is the desired inequality (2.74). The proof is complete.

Following Remark 2.2, we can obtain the following result.

Theorem 2.12. Let $\mathbb{T}$ be a time scale with $a, b \in \mathbb{T}$ and let $\alpha, \beta$ be positive real numbers such that $\alpha+\beta>1$, and let $p$, $q$ be nonnegative rd-continuous functions on $(a, b)_{\mathbb{T}}$ and $y \in C_{\mathrm{rd}}^{(n)}([a, b] \cap \mathbb{T})$. If $y^{\left(\Delta_{i}\right)}(a)=0, k \leq i \leq n-1$, then

$$
\int_{a}^{b} q(t)\left|y^{\Delta_{k}}(t)\right|^{\alpha}\left|y^{\Delta_{n}}(t)\right|^{\beta} \Delta t \leq \Lambda_{4}(a, b, \alpha, \beta) \int_{a}^{b} p(t)\left|y^{\Delta_{n}}(t)\right|^{\alpha+\beta} \Delta t
$$

where

$$
\begin{aligned}
& \Lambda_{4}(a, b, \alpha, \beta) \\
& =\left(\frac{\beta}{\alpha+\beta}\right)^{\beta /(\alpha+\beta)}\left(\int_{a}^{b} \frac{q^{(\alpha+\beta) / \alpha}(t)}{p^{\beta / \alpha}(t)}\left(\int_{a}^{t} \frac{\left(h_{n-k-1}(t, \sigma(s))\right)^{(\alpha+\beta) /(\alpha+\beta-1)}}{p^{1 /(\alpha+\beta-1)}(s)} \Delta s\right)^{(\alpha+\beta-1)} \Delta t\right)^{\alpha /(\alpha+\beta)} .
\end{aligned}
$$


Instead of (2.25), we can use the relation between $g_{n}$ and $h_{n}$ and define

$$
y(t):=(-1)^{n} \int_{t}^{b} g_{n-1}(\sigma(s), t) y^{\Delta_{n}}(s) \Delta s
$$

Proceeding as pervious by using the same arguments and using (2.90) one can obtain some results when $y^{\Delta_{i}}(b)=0$, for $i=0,1, \ldots, n-1$. For example one can get the following results.

Theorem 2.13. Letting $\mathbb{T}$ be a time scale with $a, b \in \mathbb{T}$ and $y \in C_{\mathrm{rd}}^{(n)}([X, b] \cap \mathbb{T})$. If $y^{\Delta_{i}}(b)=0$, for $i=0,1, \ldots, n-1$, then

$$
\int_{a}^{b}|y(t)|\left|y^{\Delta_{n}}(t)\right| \Delta t \leq \sqrt{\frac{1}{2}}\left(\int_{a}^{b}\left(\int_{t}^{b} g_{n-1}^{2}(\sigma(s), t) \Delta s\right) \Delta t\right)^{1 / 2} \int_{a}^{b}\left|y^{\Delta_{n}}(t)\right|^{2} \Delta t .
$$

Theorem 2.14. Let $\mathbb{T}$ be a time scale with $a, b \in \mathbb{T}$ and let $l, m$ be positive real numbers such that $l+m>1$. Let $y \in C_{\mathrm{rd}}^{(n)}([a, b] \cap \mathbb{T})$. If $y^{\Delta_{i}}(b)=0$, for $i=0,1, \ldots, n-1$, then

$$
\begin{aligned}
& \int_{a}^{b}|y(t)|^{l}\left|y^{\Delta_{n}}(t)\right|^{m} \Delta t \\
& \quad \leq\left(\int_{a}^{b}\left(\int_{t}^{b} g_{n-1}^{(l+m) /(l+m-1)}(\sigma(s), t) \Delta s\right)^{(l+m-1)} \Delta t\right)^{l /(l+m)} \int_{a}^{b}\left|y^{\Delta_{n}}(t)\right|^{l+m} \Delta t .
\end{aligned}
$$

Remark 2.15. Similar results as in Theorems 2.13 and 2.14 can be obtained from the results in the rest of the paper, but in this case one will use $y^{\Delta_{i}}(b)=0$, for $i=0,1, \ldots, n-1$, instead of If $y^{\Delta_{i}}(a)=0$, for $i=0,1, \ldots, n-1$, and $g_{n}(s, t)$ instead of $h_{n}(t, s)$.

Remark 2.16. It is worth mentioning here that the results in this paper can be used to derive some inequalities on different time scales based on the definition of the corresponding function $h_{k}(t, s)$.

For example if $\mathbb{T}=\mathbb{R}$, then from Corollary 2.8, Theorems 2.11 and 2.12 and using (2.13), we get the following inequalities of Opial's type in $\mathbb{R}$.

Theorem 2.17. Let $a, b \in \mathbb{R}$ and let $l, m$ be positive real numbers such that $l+m=1$, and $y \in$ $C^{(n)}([a, b] \cap \mathbb{R})$. If $y^{(i)}(a)=0, k \leq i \leq n-1$, then

$$
\int_{a}^{b}\left|y^{(k)}(t)\right|^{l}\left|y^{(n)}(t)\right|^{m} d t \leq \frac{m^{m}(b-a)^{l(n-k)}}{((n-k) !)^{l}}\left(\int_{a}^{b}\left|y^{(n)}(t)\right| d t\right) .
$$


Theorem 2.18. Let $a, b \in \mathbb{R}$ and let $\alpha, \beta$ be positive real numbers such that $\alpha+\beta>1$, and let $p, q$ be nonnegative continuous functions on $(a, b)$ and $y \in C^{(n)}([a, b] \cap \mathbb{R})$. If $y^{(i)}(a)=0$, for $i=0,1, \ldots, n-1$, then

$$
\int_{a}^{b} q(t)|y(t)|^{\alpha}\left|y^{\Delta_{n}}(t)\right|^{\beta} d t \leq \Lambda_{5}(a, b, \alpha, \beta) \int_{a}^{b} p(t)\left|y^{\Delta_{n}}(t)\right|^{\alpha+\beta} d t
$$

where

$$
\begin{aligned}
& \Lambda_{5}(a, b, \alpha, \beta) \\
& \quad=\left(\frac{\beta}{\alpha+\beta}\right)^{\beta /(\alpha+\beta)}\left(\int_{a}^{b} \frac{q^{(\alpha+\beta) / \alpha}(t)}{p^{\beta / \alpha}(t)}\left(\int_{a}^{t} \frac{(t-s)^{(n-1)(\alpha+\beta) /(\alpha+\beta-1)}}{(n-1) ! p^{1 /(\alpha+\beta-1)}(s)} d s\right)^{(\alpha+\beta-1)} d t\right)^{\alpha /(\alpha+\beta)} .
\end{aligned}
$$

Theorem 2.19. Let $a, b \in \mathbb{R}$ and let $\alpha, \beta$ be positive real numbers such that $\alpha+\beta>1$, and let $p, q$ be nonnegative continuous functions on $(a, b)$ and $y \in C^{(n)}([a, b] \cap \mathbb{R})$. If $y^{(i)}(a)=0, k \leq i \leq n-1$, then

$$
\int_{a}^{b} q(t)\left|y^{(k)}(t)\right|^{\alpha}\left|y^{(n)}(t)\right|^{\beta} d t \leq \Lambda_{6}(a, b, \alpha, \beta) \int_{a}^{b} p(t)\left|y^{(n)}(t)\right|^{\alpha+\beta} d t
$$

where

$$
\begin{aligned}
\Lambda_{6}(a, b, \alpha, \beta)= & \left(\frac{\beta}{(\alpha+\beta)}\right)^{\beta /(\alpha+\beta)}\left(\frac{1}{(n-k-1) !}\right)^{\beta /(\alpha+\beta)} \\
& \times\left(\int_{a}^{b} \frac{q^{(\alpha+\beta) / \alpha}(t)}{p^{\beta / \alpha}(t)}\left(\int_{a}^{t} \frac{(t-s)^{(n-k-1)(\alpha+\beta) /(\alpha+\beta-1)}}{p^{1 /(\alpha+\beta-1)}(s)} d s\right)^{(\alpha+\beta-1)} d t\right)^{\alpha /(\alpha+\beta)} .
\end{aligned}
$$

When $\mathbb{T}=\mathbb{Z}$, we have $y^{\Delta}(t)=\Delta y(t)=y(t+1)-y(t)$ and $\Delta^{(i)}=\Delta\left(\Delta^{(i-1)}\right)$. Using the fact that $h_{k}(t, s) \leq(t-s)^{k} / k$ !, we get from Corollary 2.8 and Theorems 2.11 and 2.12 the following discrete inequalities.

Theorem 2.20. Let $a, b \in \mathbb{N}$ and let $l, m$ be positive real numbers such that $l+m=1$. If $\Delta^{(i)} y(a)=0$, $k \leq i \leq n-1$, then

$$
\sum_{t=a}^{b-1}\left|\Delta^{(k)} y(t)\right|^{l}\left|\Delta^{(n)} y(t)\right|^{m} \leq \frac{m^{m}(b-a)^{l(n-k)}}{((n-k) !)^{l}} \sum_{t=a}^{b-1}\left|\Delta^{(n)} y(t)\right|
$$


Theorem 2.21. Let $a, b \in \mathbb{N}$ and let $\alpha, \beta$ be positive real numbers such that $\alpha+\beta>1$, and let $p, q$ be nonnegative sequences. If $\Delta^{(i)} y(a)=0$, for $i=0,1, \ldots, n-1$, then

$$
\sum_{t=a}^{b-1} q(t)|y(t)|^{\alpha}\left|\Delta^{(n)} y(t)\right|^{\beta} \leq \Lambda_{7}(a, b, \alpha, \beta) \sum_{t=a}^{b-1} p(t)\left|\Delta^{(n)} y(t)\right|^{\alpha+\beta}
$$

where

$$
\begin{aligned}
& \Lambda_{7}(a, b, \alpha, \beta) \\
& \quad=\left(\frac{\beta}{\alpha+\beta}\right)^{\beta /(\alpha+\beta)}\left(\sum_{t=a}^{b-1} \frac{q^{(\alpha+\beta) / \alpha}(t)}{p^{\beta / \alpha}(t)}\left(\sum_{s=a}^{t-1} \frac{(t-s)^{(n-1)(\alpha+\beta) /(\alpha+\beta-1)}}{(n-1) ! p^{1 /(\alpha+\beta-1)}(s)}\right)^{(\alpha+\beta-1)}\right)^{\alpha /(\alpha+\beta)} .
\end{aligned}
$$

Theorem 2.22. Let $a, b \in \mathbb{N}$ and let $\alpha, \beta$ be positive real numbers such that $\alpha+\beta>1$, and let $p, q$ be nonnegative sequences. If $\Delta^{(i)} y(a)=0,0 \leq k \leq i \leq n-1$, then

$$
\sum_{t=a}^{b-1} q(t)\left|\Delta^{(k)} y(t)\right|^{\alpha}\left|\Delta^{(n)} y(t)\right|^{\beta} d t \leq \Lambda_{8}(a, b, \alpha, \beta) \sum_{t=a}^{b-1} p(t)\left|\Delta^{(n)} y(t)\right|^{\alpha+\beta}
$$

where

$$
\begin{aligned}
\Lambda_{8}(a, b, \alpha, \beta)= & \left(\frac{\beta}{(\alpha+\beta)}\right)^{\beta /(\alpha+\beta)}\left(\frac{1}{(n-k-1) !}\right)^{\beta /(\alpha+\beta)} \\
& \times\left(\sum_{t=a}^{b-1} \frac{q^{(\alpha+\beta) / \alpha}(t)}{p^{\beta / \alpha}(t)}\left(\sum_{s=a}^{t-1} \frac{(t-s)^{(n-k-1)(\alpha+\beta) /(\alpha+\beta-1)}}{p^{1 /(\alpha+\beta-1)}(s)}\right)^{(\alpha+\beta-1)}\right)^{\alpha /(\alpha+\beta)} .
\end{aligned}
$$

Similar results when $\mathbb{T}=h \mathbb{Z}$ and $\mathbb{T}=q^{\mathbb{N}_{0}}=\left\{q^{t}: t \in \mathbb{N}_{0}\right\}$ where $q>1$ and different time scales can be obtained as in Theorems 2.17 and 2.22. The details are left to the reader.

Problem 1. It will be interesting to extend the pervious results and prove some inequalities of the form

$$
\int_{a}^{X} q(t)\left|y^{\Delta_{k}}(t)\right|^{\alpha}\left|y^{\Delta_{k+1}}(t)\right|^{\beta} \Delta t \leq \Lambda(a, b, \alpha, \beta) \int_{a}^{t} p(t)\left|y^{\Delta_{n}}(t)\right|^{\alpha+p} \Delta t
$$

where $\Lambda$ is the constant of the inequality that needs to be determined.

\section{References}

[1] B. Karpuz, B. Kaymakçalan, and Ö. Öcalan, “A generalization of Opial's inequality and applications to second-order dynamic equations," Differential Equations and Dynamical Systems, vol. 18, no. 1-2, pp. $11-18,2010$. 
[2] S. H. Saker, "Opial's type inequalities on time scales and some applications," Annales Polonici Mathematici, vol. 104, pp. 243-260, 2012.

[3] S. H. Saker, "New inequalities of Opial's type on time scales and some of their applications," Discrete Dynamics in Nature and Society, vol. 2012, Article ID 362526, 23 pages, 2012.

[4] V. Kac and P. Cheung, Quantum Calculus, Springer, New York, NY, USA, 2002.

[5] V. Spedding, "Taming Nature's Numbers, New Scientist," pp. 28-31, 2003.

[6] S. H. Saker, "Applications of Wirtinger inequalities on the distribution of zeros of the Riemann zetafunction," Journal of Inequalities and Applications, vol. 2010, Article ID 215416, 15 pages, 2010.

[7] S. H. Saker, "Applications of Opial and Wirtinger inequalities on zeros of third order differential equations," Dynamic Systems and Applications, vol. 20, no. 4, pp. 479-494, 2011.

[8] S. H. Saker, "Lyapunov type inequalities for a second order differential equation with a damping term," Annales Polonici Mathematici, vol. 103, no. 1, pp. 37-57, 2012.

[9] S. H. Saker, "Lyapunov inequalities for half-linear dynamic equations on time scales and disconjugacy," Dynamics of Continuous, Discrete E Impulsive Systems B, vol. 18, no. 2, pp. 149-161, 2011.

[10] G. A. Anastassiou, "Time scales inequalities," International Journal of Difference Equations, vol. 5, no. 1, pp. 1-23, 2010.

[11] B. Karpuz and U. M. Özkan, "Some generalizations for Opial's inequality involving several functions and their derivatives of arbitrary order on arbitrary time scales," Mathematical Inequalities $\mathcal{E}$ Applications, vol. 14, no. 1, pp. 79-92, 2011.

[12] H. M. Srivastava, K. L. Tseng, S. J. Tseng, and J. C. Lo, “Some generalizations of Maroni's inequality on time scales," Mathematical Inequalities E Applications, vol. 14, no. 2, pp. 469-480, 2011.

[13] F. H. Wong, W. C. Lian, S. L. Yu, and C. C. Yeh, "Some generalizations of Opial's inequalities on time scales," Taiwanese Journal of Mathematics, vol. 12, no. 2, pp. 463-471, 2008.

[14] R. P. Agarwal, M. Bohner, D. O’Regan, and S. H. Saker, "Some dynamic Wirtinger-type inequalities and their applications," Pacific Journal of Mathematics, vol. 252, no. 1, pp. 1-18, 2011.

[15] M. Bohner and B. Kaymakçalan, "Opial inequalities on time scales," Annales Polonici Mathematici, vol. 77, no. 1, pp. 11-20, 2001.

[16] S. H. Saker, "Some Opial-type inequalities on time scales," Abstract and Applied Analysis, vol. 2011, Article ID 265316, 19 pages, 2011.

[17] H. M. Srivastava, K. L. Tseng, S. J. Tseng, and J. C. Lo, "Some weighted Opial-type inequalities on time scales," Taiwanese Journal of Mathematics, vol. 14, no. 1, pp. 107-122, 2010.

[18] M. Bohner and A. Peterson, Dynamic Equations on Time Scales: An Introduction with Applications, Birkhäuser, Boston, Mass, USA, 2001.

[19] M. Bohner and A. Peterson, Eds., Advances in Dynamic Equations on Time Scales, Birkhäuser, Boston, Mass, USA, 2003.

[20] S. Hilger, "Analysis on measure chains-a unified approach to continuous and discrete calculus," Results in Mathematics, vol. 18, no. 1-2, pp. 18-56, 1990.

[21] R. P. Agarwal and M. Bohner, "Basic calculus on time scales and some of its applications," Results in Mathematics, vol. 35, no. 1-2, pp. 3-22, 1999.

[22] W. G. Kelley and A. C. Peterson, Difference Equations, An Introduction with Applications, Academic Press, New York, NY, USA, 1991.

[23] R. J. Higgins and A. Peterson, "Cauchy functions and Taylor's formula for time scales T," in Proceedings of the 6th International Conference on Difference Equations, pp. 299-308, CRC, Boca Raton, Fla, USA, 2004. 


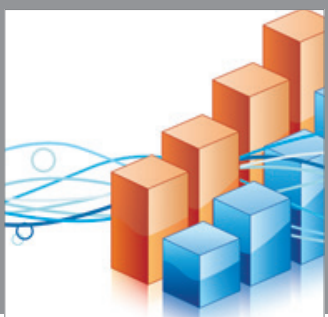

Advances in

Operations Research

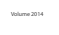

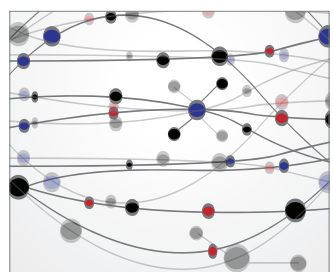

\section{The Scientific} World Journal
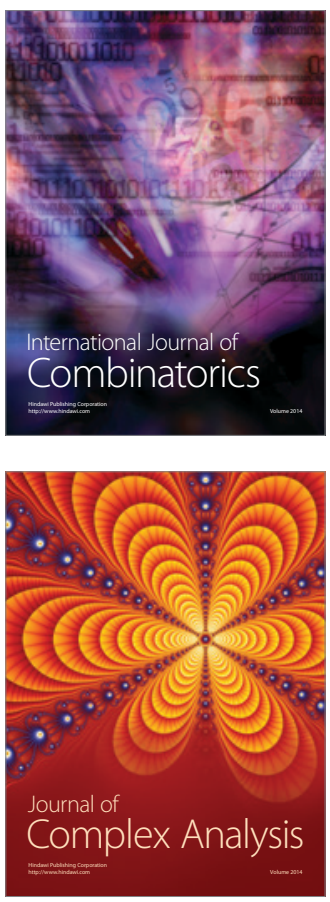

International Journal of

Mathematics and

Mathematical

Sciences
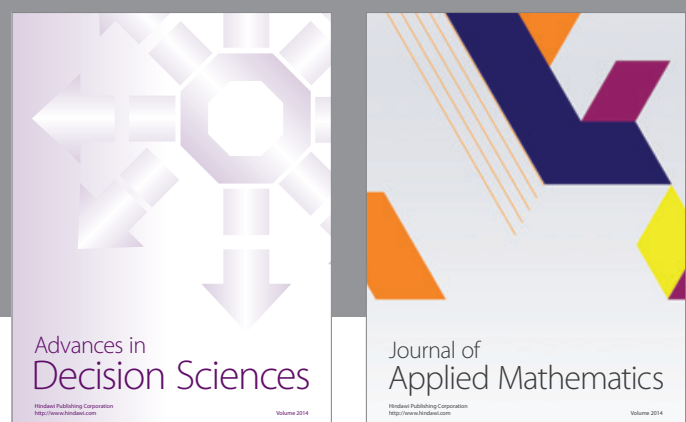

Journal of

Applied Mathematics
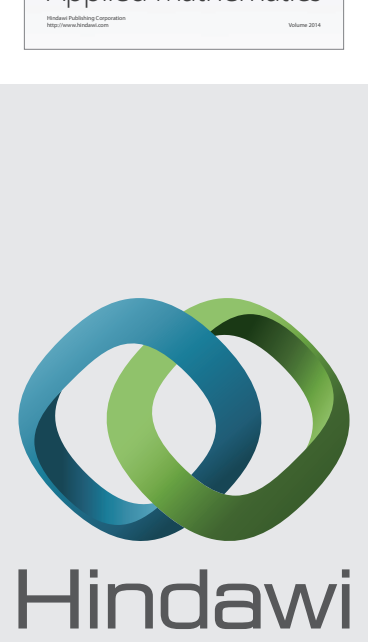

Submit your manuscripts at http://www.hindawi.com
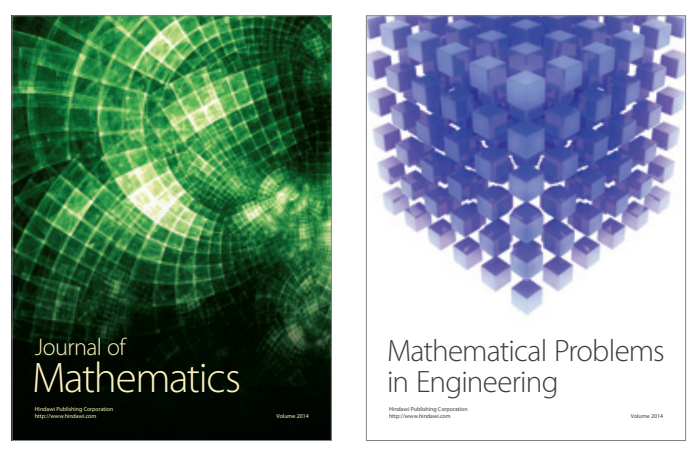

Mathematical Problems in Engineering
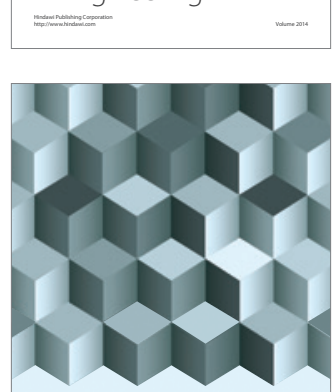

Journal of

Function Spaces
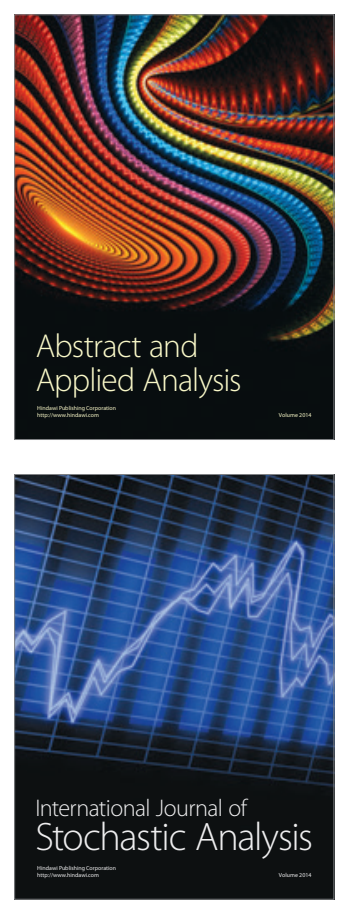

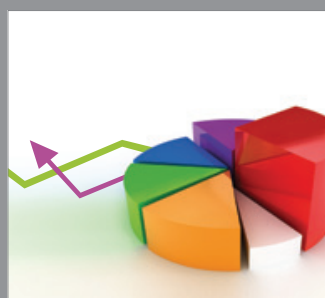

ournal of

Probability and Statistics

Promensencen
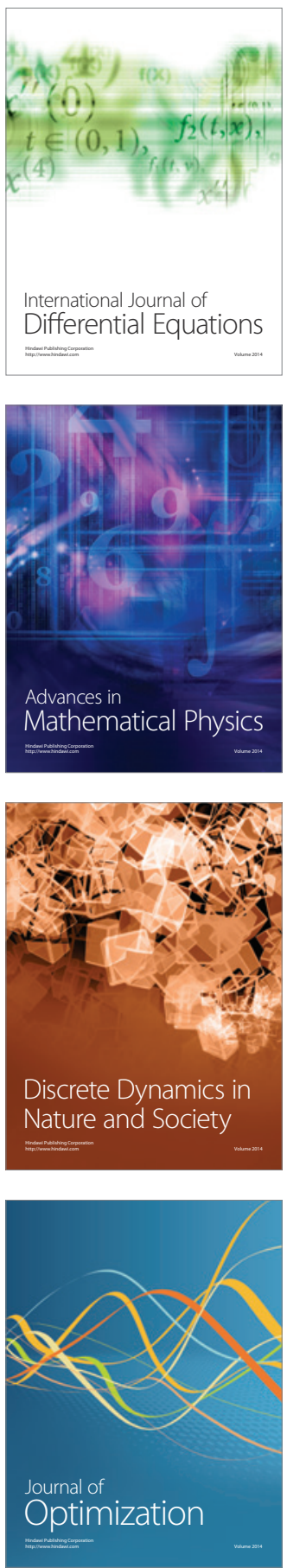